\title{
Scenario for the renormalization in the 4D Yang-Mills theory
}

\author{
L. D. Faddeev \\ St. Petersburg dept. of Steklov \\ Mathematical Institute \\ and St. Petersburg University
}

Yang-Mills quantum field theory has unique character, allowing a selfconsistent formulation in the four dimensional space-time. Two important properties - asymptotic freedom and dimensional transmutation — are characteristic features of this theory, which distinguish it from others. I think, that the typical textbook exposition of this theory, based on general paradigm of QFT, still does not underline these specifics. In my talk I propose a scheme for the description of the Yang-Mills theory which exactly does this. I do not claim finding anything new, my proposal has simply a methodological value.

As a main tool for my presentation I have chosen the object, called the effective action, defined via the background field. Following Feynman ideas I consider the functional of a background field as the generating functional for the $S$-matrix, whereas the Schwinger functional of external current, generating the Green functions, needs LSZ reduction formulas to define $S$-matrix. Moreover, the latter functional is not manifestly gauge invariant.

Ironically the standard description of the background field method [1], [2], 3] uses the external current and Legendre transformation. Alternative formulation began in [4] and entering [5] was improved in [6]. In what follows I use the latter approach.

An effective action $W(B)$ is a functional of a classical Yang-Mills field $B_{\mu}(x)$ given by a series in a dimensionless coupling $\alpha$

$$
W(B)=\frac{1}{\alpha} W_{-1}(B)+W_{0}(B)+\sum_{n \geq 1} \alpha^{n} W_{n}(B),
$$

where $W_{-1}(B)$ is a classical action, $W_{0}(B)$ is a one loop correction, defined via the determinants of vector and scalar operators $M_{1}$ and $M_{0}$

$$
M_{0}=\nabla_{\mu}^{2}, \quad M_{1}=\nabla_{\sigma}^{2} \delta_{\mu \nu}+2\left[F_{\mu \nu}, \cdot\right]
$$


where

$$
\begin{gathered}
\nabla_{\mu}=\partial_{\mu}+B_{\mu}, \\
F_{\mu \nu}=\partial_{\mu} B_{\nu}-\partial_{\nu} B_{\mu}+\left[B_{\mu}, B_{\nu}\right] . \\
W_{0}=-\frac{1}{2} \ln \operatorname{det} M_{1}+\ln \operatorname{det} M_{0}
\end{gathered}
$$

and $W_{k}, k=1,2 \ldots$ are defined as the contribution of strongly connected vacuum diagramms with $k+1$ loops, constructed via Green functions $M_{0}^{-1}$ and $M_{1}^{-1}$ and vertices defined by the forms of vector and scalar fields $a_{\mu}(x)$, $\bar{c}(x), c(x)$

$$
\begin{aligned}
\Gamma_{3}(B) & =g \int \operatorname{tr} \nabla_{\mu} a_{\nu}\left[a_{\mu}, a_{\nu}\right] d^{4} x, \\
\Gamma_{4}(B) & =g^{2} \int \operatorname{tr}\left[a_{\mu}, a_{\nu}\right]^{2} d^{4} x, \\
\Omega(B) & =g \int \operatorname{tr} \nabla_{\mu} \bar{c}\left[a_{\mu}, c\right] d^{4} x,
\end{aligned}
$$

where $g=\frac{1}{2} \sqrt{\alpha}$, taking into account anticommuting propeties of ghosts $\bar{c}(x)$, $c(x)$.

The divergences of the diagramms should be regularized. I believe that there exists a regularization, defined by the cut-off momentum $\Lambda$ such that all infinities are powers in

$$
L=\ln \frac{\Lambda}{\mu}
$$

where $\mu$ is some normalization mass. Unfortunately presently I do not know a satisfactory procedure for such a regularization. That is why I call my exposition a "scenario".

The renormalizability of the Yang-Mills theory means, that there exists a dependence of the coupling constant $\alpha$ on cutoff $\Lambda$ such that the full action $W(B)$ is finite.

In the case of one loop everything is clear. The functional $W_{0}(B)$ can be defined via the proper time method of Fock [7] giving formula

$$
W_{0}(B)=\int_{0}^{\infty} \frac{d s}{s} T(B, s),
$$

where the functional $T(B, s)$ has the following behavior for small $s$

$$
T(B, s)=T_{0}(B)+s T_{1}(B, s)
$$


and

$$
T_{0}(B)=\frac{1}{2} \beta_{1} W_{-1},
$$

where $\beta_{1}$ is a famous negative constant. So the only divergence is proportional to the classical action and can be compensated by the renormalization of the coupling constant $\alpha$. In more detail, we regularize $W_{0}(B)$ as follows

$$
W_{0}^{\mathrm{reg}}(B)=\int_{0}^{1 / \Lambda^{2}} d s T_{1}(B, s)+\int_{1 / \Lambda^{2}}^{\infty} \frac{d s}{s} T(B, s)
$$

and choose the dependence of the coupling constant $\alpha$ on $\Lambda$ as

$$
\frac{1}{\alpha(\Lambda)}=-\beta_{1} \ln \frac{\Lambda}{m}
$$

where $m$ is a new parameter with the dimension of mass. It is clear, that the regularized one loop $W(B)$ does not depend on $\Lambda$ and so is finite. More explicitly, we rewrite $W_{0}^{\text {reg }}(B)$ as follows

$$
W_{00}(B)=W_{00}+W_{01} L
$$

where

$$
W_{0}^{\mathrm{reg}}(B)=\int_{0}^{1 / \mu^{2}} d s T_{1}(B, s)+\int_{1 / \mu^{2}}^{\infty} \frac{d s}{s} T(B, s)
$$

and

$$
W_{01}=\beta_{1} W_{-1}
$$

and define the renormalized running coupling constant

$$
\frac{1}{\alpha(\mu)}=-\beta_{1} \ln \frac{\mu}{m}
$$

so that

$$
W_{1 \mathrm{loop}}^{\mathrm{reg}}(B)=\frac{1}{\alpha(\mu)} W_{-1}+W_{00} .
$$

Thus we have traded the dimensionless $\alpha$ for a dimensional parameter $m$. However $m$ enters trivially, defining the scale. Observe that $\alpha(\Lambda)$ and $\alpha(\mu)$ are the values of the same function for two values of the argument. This function satisfies the first approximation to Gell-Mann Low equation

$$
x \frac{d}{d x} \alpha(x)=\beta(\alpha)=\beta_{1} \alpha^{2},
$$


where the RHS does not depend on $x$ and $m$ plays the role of the conserved integral. The shift of $x$ can be interpreted as an abelian group action, this is the famous renormalization group. The same is true for the whole one loop functional

$$
W_{1 \text { loop }}^{\text {reg }}(B, \Lambda)=W_{1 \text { loop }}(B, \mu)
$$

and the renormalized action $W(B, \mu)$ does not depend on the running momentum $\mu$.

My scenario is based on the assumption that the whole $W(B)$ has the same properties. I believe that all infinities in $W(B)$ can be combined into the form

$$
\begin{aligned}
W(B, \Lambda)= & \frac{1}{\alpha} W_{-1}+W_{00}+W_{01} L+ \\
& +\alpha\left(W_{10}+W_{11} L\right)+\ldots+ \\
& +\alpha^{n}\left(W_{n 0}+W_{n 1} L+\ldots W_{n n} L^{n}\right)+\ldots
\end{aligned}
$$

where the functionals $W_{k 0}(B), k=0, \ldots$ are finite and depend on $\mu$. The coupling constant $\alpha(\Lambda)$ should satisfy the full Gell-Mann Low equation

$$
\Lambda \frac{d \alpha}{d \Lambda}=\beta(\alpha)=\beta_{1} \alpha^{2}+\beta_{2} \alpha^{3}+\ldots \beta_{n} \alpha^{n}+\ldots
$$

and the full $W(B)$ should be independent of $L$ and so it is finite.

The equation

$$
\frac{d W}{d L}=0
$$

immediately gives

$$
W_{11}=\beta_{2} W_{-1}
$$

and leads to an equation, expressed via double series in powers of $\alpha$ and $L$. The condition that the corresponding coefficients vanish leads to the recurrent relations expressing $W_{n m}$ via $W_{n-1, m}, n=2,3, \ldots, n \geq m$.

Here are examples of such equations at the lowest orders

$$
\begin{aligned}
& \beta_{1} W_{10}+W_{21}=\beta_{3} W_{-1} \\
& \beta_{2} W_{10}+2 \beta_{1} W_{20}+W_{31}=\beta_{4} W_{-1} \\
& \beta_{1} W_{11}+2 W_{22}=0 \\
& \beta_{2} W_{11}+2 \beta_{1} W_{21}+2 W_{32}=0 .
\end{aligned}
$$


One can solve some of these equations exactly. For instance for the highest coefficients $W_{n n}$ we get relation

$$
(n-1) \beta_{1} W_{n-1, n-1}+n W_{n n}=0
$$

and as $W_{11}$ is proportional to $W_{-1}$ the same is true for all $W_{n n}$. Thus their contribution $\sum \alpha^{n} L^{n} W_{n n}$ is summed up to

$$
\frac{\beta_{2}}{\beta_{1}} \ln \left(1+\beta_{1} \alpha L\right) W_{-1}
$$

and this gives the next correction to the coefficient in front of $W_{-1}$ in $W(B)$, namely

$$
\frac{1}{\alpha}+\beta_{1} L+\frac{\beta_{2}}{\beta_{1}}\left[\ln \alpha+\ln \left(\frac{1}{\alpha}+\beta_{1} L\right)\right]
$$

leading to the next approximation for the renormalizaed coupling constant

$$
\frac{1}{\alpha_{r}}=\frac{1}{\alpha}+\beta_{1} L+\frac{\beta_{2}}{\beta_{1}} \ln L
$$

consistent with the Gell-Mann Low equation.

We see that all coefficients $W_{n m}, n \geq m, n \geq 1$ are expressed via the finite ones $W_{n 0}$. More detailed investigation of these equations [8] shows, that the full expression for $W(B)$ can be rewritten in terms of $W_{n 0}$ and powers of renormalized coupling constant

$$
W(B, \mu)=\frac{1}{\alpha_{r}} W_{-1}+W_{00}+\sum \alpha_{r}^{n} W_{n 0} .
$$

This is consistent with the equation

$$
W_{\text {reg }}(B, \Lambda)=W(B, \mu)
$$

and

$$
\left.L\right|_{\Lambda=\mu}=0 .
$$

Let us comment, that the recurrence relations make sense when the only nonzero coefficients in $\beta$-function are $\beta_{1}$ and $\beta_{2}$. This makes possible to speculate, that there exist the regularizations in which $\beta_{3}=\beta_{4}=\ldots=0$. 


\section{Acknowledgments}

This work is partially supported by RFBR grant 12-01-00207 and the programme "Mathematical problems of nonlinear dynamics" of RAS. I thank

S. Derkachov and A. Ivanov for collaboration in development of my programm.

\section{References}

[1] B. S. DeWitt, "Quantum Theory of Gravity. 2. The Manifestly Covariant Theory," Phys. Rev. 162 (1967) 1195.

B. S. DeWitt, "Quantum Theory of Gravity. 3. Applications of the Covariant Theory," Phys. Rev. 162 (1967) 1239.

[2] G. 't Hooft, "The Background Field Method in Gauge Field Theories," In *Karpacz 1975, Proceedings, Acta Universitatis Wratislaviensis No.368, Vol.1*, Wroclaw 1976, 345-369.

[3] L. F. Abbott, "Introduction to the Background Field Method," Acta Phys. Polon. B 13 (1982) 33.

[4] I. Y. Arefeva, L. D. Faddeev and A. A. Slavnov, "Generating Functional for the s Matrix in Gauge Theories," Theor. Math. Phys. 21 (1975) 1165 [Teor. Mat. Fiz. 21 (1974) 311].

[5] L. D. Faddeev and A. A. Slavnov, "Gauge Fields. Introduction To Quantum Theory," Front. Phys. 50 (1980) 1, [Front. Phys. 83 (1990) 1].

[6] L. D. Faddeev, "Mass in Quantum Yang-Mills Theory: Comment on a Clay Millenium problem," arXiv:0911.1013 [math-ph].

[7] V. Fock, "Proper time in classical and quantum mechanics," Phys. Z. Sowjetunion 12 (1937) 404.

[8] S. Derkachov, L. Faddeev, A. Ivanov, to be published. 